FERMILAB-Conf-97/155

\title{
Synchrotron Radiation Interferences Between Small Dipoles at LEP
}

\author{
C. Bovet, A. Burns, F. Méot, M. Placidi, E. Rossa and J. de Vries \\ CERN \\ Geneva, Switzerland
}

Fermi National Accelerator Laboratory

P.O. Box 500, Batavia, Illinois 60510

June 1997

Presented at the Particle Accelerator Conference PAC'97,

Vancouver, Canada, May 12-16, 1997 


\section{Disclaimer}

This report was prepared as an account of work sponsored by an agency of the United States Government. Neither the United States Government nor any agency thereof, nor any of their employees, makes any warranty, expressed or implied, or assumes any legal liability or responsibility for the accuracy, completeness, or usefulness of any information, apparatus, product, or process disclosed, or represents that its use would not infringe privately owned rights. Reference herein to any specific commercial product, process, or service by trade name, trademark, manufacturer, or otherwise, does not necessarily constitute or imply its endorsement, recommendation, or favoring by the United States Government or any agency thereof. The views and opinions of authors expressed herein do not necessarily state or reflect those of the United States Government or any agency thereof.

\section{Distribution}

Approved for public release; further dissemination unlimited. 


\title{
SYNCHROTRON RADIATION INTERFERENCES BETWEEN SMALL DIPOLES AT LEP
}

\author{
C. Bovet, A. Burns, F. Méot*, M. Placidi, E. Rossa, J. de Vries \\ CERN, Geneva, Switzerland.
}

\begin{abstract}
Synchrotron Radiation interferences between small dipoles in the very low (visible) frequency range have been studied at the LEP diagnostic mini-wiggler. Their understanding allowed a substantial brightness gain by adequate layout modifications. The phenomenon is described analytically in terms of time coherence effects. This serves as a basis for further detailed numerical simulations of the experiment by means of stepwise ray-tracing, and allows precise interpretation of the spectral, polarization and intensity measurements collected at LEP. It also provides guidelines for SR diagnostic at injection energy in LHC.
\end{abstract}

\section{INTRODUCTION}

Two identical devices (mini-wigglers) [1] provide dedicated SR for transverse and longitudinal bunch profile measurements on $\mathrm{e}^{+}$and $\mathrm{e}^{-}$beams in LEP with a streak camera sensitive in the near-UV to visible range [2]. They are located at $\sim 67 \mathrm{~m}$ on either side of IP1 where the light beams are extracted by plane mirrors.

A first configuration exploited until 1993 (Fig. 1) involved $\mathrm{a} \sim 3 \mathrm{~m}$ long localised closed orbit bump. It was subject to harmful multiple image formation and interference effects which received theoretical interpretation [3]. This led to the design of a new configuration (Fig.2) now operational and compatible with bunch train operation at LEP2 energies [4].

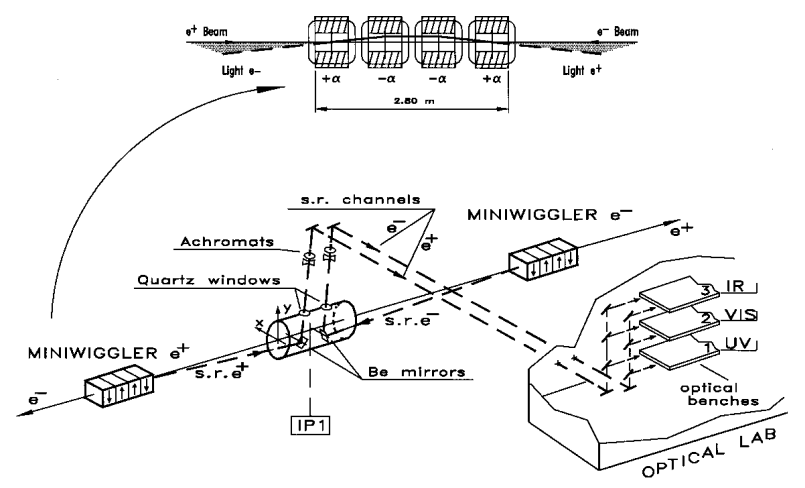

Figure 1: The first mini-wiggler set-up in the LSS1 LEP straight section. $e^{+} e^{-}$synchrotron light beams extracted at IP1 are focused in the optical laboratory.

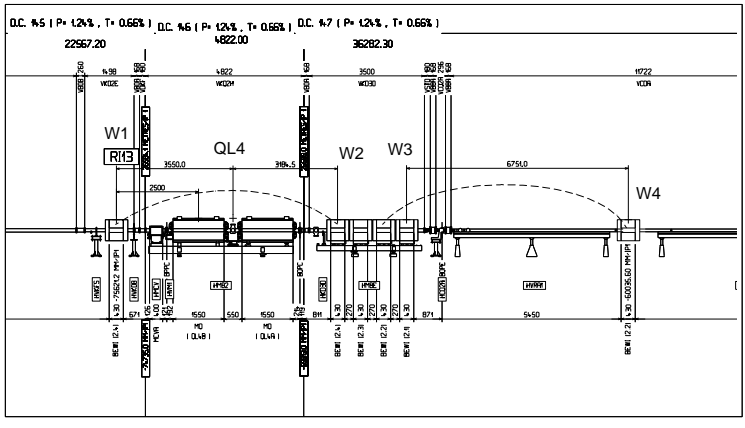

Figure 2: The new mini-wiggler layout at the left side of IP1 for diagnostics on the $e^{+}$beam. The closed orbit bump now extends over $15.6 \mathrm{~m}$ from $\mathrm{W} 1$ to $\mathrm{W} 4$. A similar set up is installed symmetrically to IP1 for the $e^{-}$beam. SR sources from both dipoles W3 and W4, now $6.75 \mathrm{~m}$ away, fall within the acceptance of the imaging optics (Fig. 1) which focuses on W4 and the effect of W3 is reduced to $\simeq 10 \%$ parasitic blurring while $\mathrm{W} 1, \mathrm{~W} 2$ are not seen.

\section{EXPERIMENTAL RESULTS}

The first comparative measurements between the original mini-wiggler set-up and a configuration where the SR emission from a single dipole could be observed were performed in 1993 [5]. In the former case the light intensity was comparable to that from the parasitic sources (e.g. the quadrupole QL4) while in the latter case about 2 orders of magnitude intensity were gained (Fig. 3). This result suggested that the interference effects could be cured on the basis of the theoretical investigations [3]. Experiments have been performed using this new configuration the main results can be summarised as follows.

The intensity from the W3-W4 pair agreed, well with SR froma single dipole confirming the remaining factor of $\sim 2$ in the interference intensity loss predicted from the numerical simulations for the case of a pair of dipoles $6 \mathrm{~m}$ apart. It is worth to mention that following the improvements in the production of visible light a series of accelerator physics experiments requiring the use of the streak camera were performed with a very low current ( $4 \mu \mathrm{A}$ single bunch).

Intensity measurements as a function of the deflection $\alpha$ in W3 and W4 were performed making use of polarising filters to separate the $\sigma$-and the $\pi$-components of the radiation (Table 1) [6]. The two vertical lobes of the $\pi$-component were clearly observed on a screen positioned slightly out of focus. This method was adopted to precisely determine the longitudinal position of the image plane defined by the superposition of the two $\pi$-lobes.

${ }^{*}$ BD/Physics, FNAL, Batavia, IL, USA. On leave from CEA/DSM, Saclay, France 


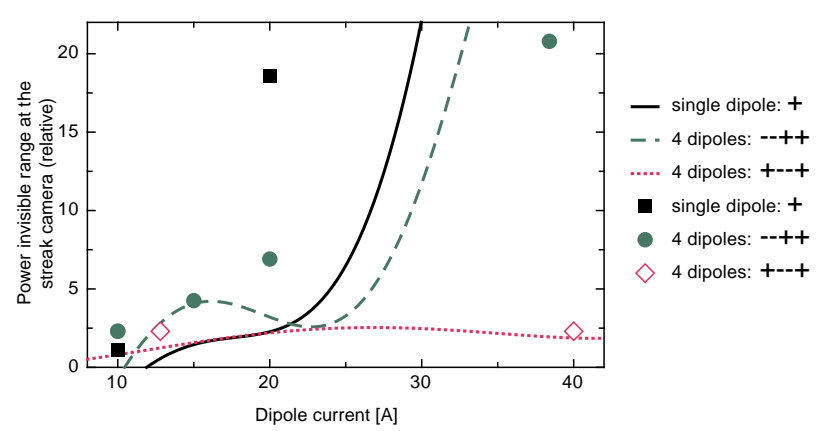

Figure 3: Comparison of the experimental data [4,5] with numerical simulations (continuous curves [3]) performed in 1993 and 1994. The radiated power at the streak camera is shown as a function of the dipole excitation for two different 4-dipole configurations and for the single-dipole case. The + and - are deflection signs in the dipoles W1-W4 juxtaposed as in Fig. 1. Note a remarkable result in the +--+ configuration: due to the interference, the SR intensity stays constant within the 10-40A current range.

Table 1: Integrated (relative) intensities of the light spots at the image plane. (Beam energy $E=20 \mathrm{GeV}$ ). In the case of the images corresponding to $40 \mathrm{~A}$ excitation it was possible to measure the W4 and W3 light spots separately. The integrals obtained for the other images correspond to a combination of the light from the 2 sources. These values compare fairly well with the theoretical predictions as shown on Fig. 5.

\begin{tabular}{|l|l|l|l|l|l|l|l|}
\hline Source & $\begin{array}{l}\text { I } \\
\text { (A) }\end{array}$ & $\begin{array}{l}\alpha \\
\text { (mrad) }\end{array}$ & $\begin{array}{l}\text { Intensity } \\
\text { in } \sigma-\end{array}$ & $\begin{array}{l}\text { Intensity } \\
\text { in } \pi-\end{array}$ & $\sigma+\pi$ & $\frac{\sigma}{\sigma+\pi}$ & $\frac{\pi}{\sigma+\pi}$ \\
\hline W3 & 40 & 1.40 & 0.80 & 0.13 & 0.93 & 0.86 & 0.14 \\
W4 & 40 & 1.40 & 0.86 & 0.20 & 1.06 & 0.81 & 0.19 \\
W3+W4 & 25 & 0.88 & 2.39 & 1.71 & 4.10 & 0.58 & 0.42 \\
W3+W4 & 12 & 0.42 & 1.53 & 0.85 & 2.38 & 0.64 & 0.36 \\
\hline
\end{tabular}

\section{THEORETICAL INTERPRETATION}

\subsection{Interference}

Numerical simulations of interference [3] by ray-tracing [7] in the first 4-dipole configurations (Fig. 1) are reported in Fig. 3. Interference consists of time coherence effects which can be summarized as follows. An electron traversing a pair of dipoles (for simplification) delivers a double electric field impulse (Fig. 4) which can be written

$$
E(t)=e(t)\left[\delta\left(t-\frac{T}{2}\right)-\delta\left(t+\frac{T}{2}\right)\right]
$$

where $e(t)$ is the single dipole impulse, $\delta$ is the Dirac distribution, $t$ is the observer time, $T=d\left(1+\gamma^{2} \Psi^{2}+\right.$ $\left.K^{2} / 2\right) /\left(2 \gamma^{2} c\right)$ is the time delay between the two impulses ( $\gamma=$ relativistic Lorentz factor, $\Psi=$ observation angle, $K=$ $\alpha \gamma, c=$ light velocity, $d=$ magnet separation).

The squared Fourier transform of $E(t)$ is homogeneous to the SR brightness at the observer and is written

$$
|\tilde{E}(\omega)|^{2}=4 \sin ^{2} \frac{\omega T}{2}|\tilde{e}(\omega)|^{2}
$$

where $|\tilde{e}(\omega)|^{2}$ describes the brightness due to one dipole, homogeneous to the $K_{1 / 3}$ or $K_{2 / 3}$ Bessel functions in regular SR. For dipoles which are $d \simeq$ metres apart (Fig. 2), and $\omega \simeq 0.5 \mathrm{eV}$ (visible light, left end of the spectrum in Fig. 4), one gets $\omega T / 2 \ll 1$ and the attenuation due to the interference amounts to $\sin ^{2}(\omega T / 2) \simeq(\omega T / 2)^{2}$.

Pulling the two dipoles $6.75 \mathrm{~m}$ apart (W3-W4 in Fig. 2) leads to an increase in $\sin ^{2}(\omega T / 2)$ by a factor $(6.75 / 0.76)^{2} \simeq 80$, consistent with the measured intensity gain obtained by going from the first miniwiggler configuration to the second one.
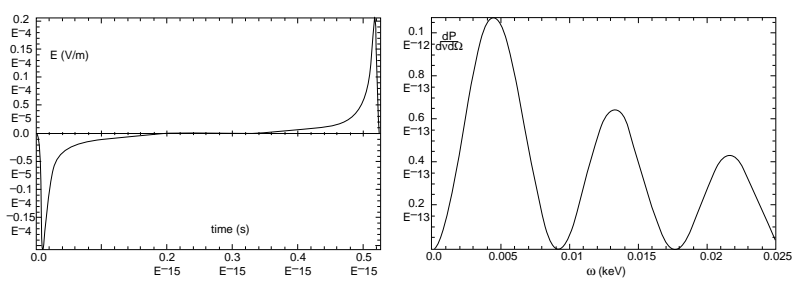

Figure 4: Double electric field impulse $E_{\sigma}(t)$ emitted by a $45 \mathrm{GeV}$ electron undergoing a $\alpha=0.43 \mathrm{mrad}$ deflection in $\mathrm{W} 3$ and $\mathrm{W} 4 \mathrm{~d}=0.76 \mathrm{~m}$ apart, and the corresponding brightness $\mathrm{d} P_{\sigma} / \mathrm{d} \nu \mathrm{d} \Omega$ (normalized to $1 \mathrm{~mA}$ circulating beam current) in the direction $\varphi=\alpha / 2, \Psi=0$.

\subsection{Single dipole}

Figure 5 presents a comparison between intensity measurements in the new configuration (Fig. 2) and the theoretical predictions schemed in Fig. 6 which displays the shape of the $\sigma$ - and $\pi$ - components of the visible SR due an electron traversing a single dipole, as observed at the surface of the extraction mirrors at IP1 (Fig. 1).

It can be shown (Fig. 5 and Table 1) that the $P_{\sigma} / P_{\pi}$ ratio varies strongly from one dipole excitation to another, but is very different from the usually assumed $(2 / 3) /(1 / 3)$ ratio of the low frequency SR approximation [9]. It can be verified that $d P_{\sigma} / d \varphi$ is zero for $\varphi=\alpha$ (Fig. 6); this is due to $\int E_{\sigma} \mathrm{dt}$ $=0$ for that particular direction of radiation [3].

An important feature of these simulations is that they involve the single SR source W4, thus neglecting any residual interference effects due to W3. Such interference would result in a distortion of the SR spectrum by a partial sinemodulation (Fig. 4) which in turn would translate into nonlinear effects in Fig. 5, where the light power as a function of the dipole excitation is implicitely correlated to the overlapping of the two sources W3 and W4.

The intensity measurements do not prove the existence of such residual interference. Nevertheless the slight discrepancies between the measurements and the simulations (Fig. 5) might reveal it. This point would deserve deeper experimental and theoretical investigations. It might for instance explain the fringes that appear in the $\pi$-spot of the W3-W4 image observed at 12A [4]. 


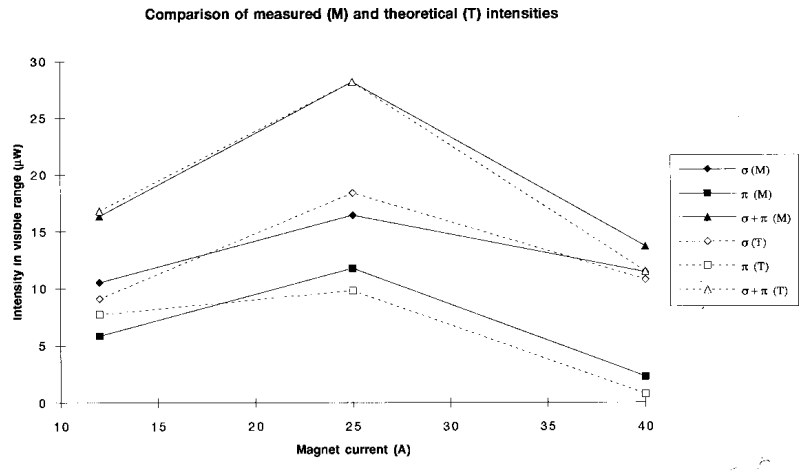

Figure 5: Comparison between the measured intensities (Table 1, $\mathrm{M}$ curves) and numerical simulations for a $20 \mathrm{GeV}$ electron, normalised to $1 \mathrm{~m} \mathrm{~A}$ beam ( $\mathrm{T}$ curves), in the $400-800 \mathrm{~nm}$ spectral range (data normalised to the calculated $\sigma+\pi$ intensity at $25 \mathrm{~A}$ ).
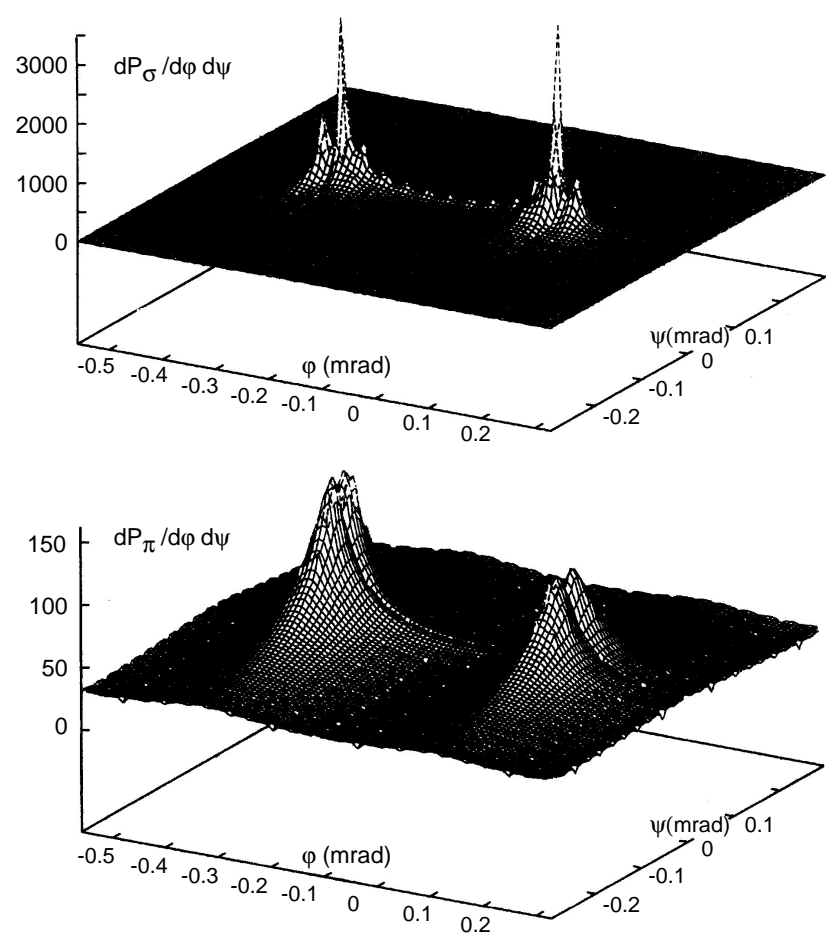

Figure 6: 3-D plot of the $\sigma$ - and $\pi$-components of the visible light intensity $(400-800 \mathrm{~nm})$ from W4 $(\alpha=-0.43 \mathrm{mrad})$, at large distance, obtained by ray-tracing of a single $20 \mathrm{GeV}$ electron [7]. The transverse axes are the angles $\varphi$ (horizontal) and $\psi$ (vertical). The end intensity peaks are due to the edge radiation [3], [8]. It can be observed that $\mathrm{d} P_{\sigma} / \mathrm{d} \varphi \mathrm{d} \psi \simeq 0$ at $\varphi=\alpha=-0.43 \mathrm{mrad}$ (upper plot).

As well, special properties apparent in Fig. 6 (such as $\int E_{\sigma} d t \neq 0$ at $\omega \simeq 0$, consistent with the concept of 'strange electromagnetic waves' as developped in Ref. [10]), deserve finer experimental investigation. The edge effect might explain the double spot shape of the $\pi$-image of W4 at 40A [4] namely the two spots corresponding to the two end peaks of W4 (Fig. 6). Works relevant with this issue have been accomplished recently [11].

\section{CONCLUSIONS}

A new configuration of the dedicated SR source (miniwiggler) compatible with LEP2 energies and the bunch train scheme is now in operation. Pulling the last two dipoles $6.75 \mathrm{~m}$ apart considerably reduces the destructive interference and improves the light intensity by a factor of $30 \mathrm{com}$ pared to the previous configuration.

These experimental results confirm the theoretical investigations and provide a deeper insight into interference issue in connection with the emission of synchrotron radiation from small dipoles. This might be of particular importance for the optimisation of LHC beam diagnostics [12] where the same ray-tracing methods and codes are used to investigate the brightness of a short dipole in the spectral range beyond the critical energy.

\section{REFERENCES}

[1] C. Bovet, M. Placidi, A dedicated synchrotron radiation source for LEP beam diagnostics, LEP Note 532, CERN, $11 / 4 / 85$

[2] E. Rossa, F. Tecker, J.C. Mathae, Performance limits of a streak camera in real time three-dimensional measurement of bunch oscillation in LEP, Preprint of 1993 PAC, May 17-20, Washington, D.C.

[3] F. Méot, Synchrotron radiation interferences at the LEP mini-wiggler, CERN SL/94-22 (AP), CERN, 28/06/94.

[4] C. Bovet, A. Burns, F. Méot, M. Placidi, E. Rossa, I. de Vries, The new configuration of the synchrotron radiation service for beam diagnostics in LEP Report SL/BI, CERN, to be published.

[5] K. Hanke, Measurements of the bunch length of LEP with a streak camera and comparison with results from LEP experiments, CERN SL/Note 95-65 (BI), CERN, 13/06/95.

[6] A.J. Burns, Measurement of intensity of synchrotron light spots for different mini-wiggler currents, CERN SL/Note 96-53 (BI), CERN, 21/08/96.

[7] F. Méot and S. Valéro, ZGOUBI users' guide, Report LNS/GT/93-12, CEA, Saclay, 1993.

[8] O. Chubar, Measurement of effective electron beam emittance using edge radiation, Kurtchatov Institute, preprint AE-5698/14 (1994).

[9] A. Hofmann, Theory of synchrotron radiation, SLAC report, SSRL-ACD Note 38, Sept. 1986, p. 32.

[10] E.G. Bessonov, On a class of electromagnetic waves, Sov. Phys. JETP53(3), March 1981.

[11] C. Denise, Ph.D. Thesis, ESRF, Grenoble, (1997).

[12] J. Bosser, C. Bovet, R. Jung, LHC Note 192, CERN, 17/6/92. 